G MC struct ure consi der i ng communi cat i on del ay and i ts appl i cat i on to mechat roni $c$ syst em

\begin{tabular}{|c|c|}
\hline 著者 & Namer i kawa Tor u, M yakawa Juni chi \\
\hline $\begin{array}{l}\text { jour nal or } \\
\text { publ i cat i on title }\end{array}$ & Proceedi ngs of the Ameri can Control Conf er ence \\
\hline number & 4282617 \\
\hline page $r$ ange & 1532- 1537 \\
\hline year & 2007-01- 01 \\
\hline URL & ht t p: //hdl . handl e. net /2297/19633 \\
\hline
\end{tabular}




\title{
GIMC Structure Considering Communication Delay and Its Application to Mechatronic System
}

\author{
Toru Namerikawa and Junichi Miyakawa
}

\begin{abstract}
This paper deals with a new GIMC structure considering a communication delay by using the Smith Predictor and its application to a mechatronic system. First, we stabilize the unstable mechatronic system by a PD controller and define the stabilized system as a new augmented plant. We design the proposed GIMC structure using the Smith Predictor based on $\mathscr{H}_{\infty}$ controllers for the new stabilized augmented plant. Finally, the proposed structure is evaluated experimentally and the effectiveness of the proposed approach is proven. In addition, we apply the proposed structure to a virtual networked control mechatronic system.
\end{abstract}

\section{INTRODUCTION}

It is well-known that general control architectures cannot achieve both performance and robustness simultaneously, because there is a tradeoff in these two specifications [1]. The Generalized Internal Model Control (GIMC) structure was proposed for this tradeoff problem [2], [3]. It can achieve both performance and robustness specifications based on a switching strategy: a nominal high performance controller $K_{0}$ controls the nominal plant and a robust controller $K$ maintains stability for perturbed plants.

Consequently, this structure has been applied to gyroscope and motor control so far, and experimentally it achieves stability in a plant perturbed by changes such as sensor failure [4], [5].

The GIMC structure is not affected by a small communication delay because this structure has high robustness. But this structure does not have been considered for larger delays such as those found in network communications[6].

It is difficult to manage a general control architecture which has a time delay. An output after delay time is predicted to use an architecture which has a plant model and a time-delayed model in the internal control loop. The Smith Predictor is well-known as an effective control method based on a predictive output [7] [8]. A control system with the Smith Predictor can be designed without considering delay because the output of the plant after delay time is canceled by the predictive output.

Our goal is to apply a new GIMC structure, considering communication delays by using the Smith Predictor, to a magnetic suspension system, which is an unstable mechatronic system, and evaluate its effectiveness via control experiments. In addition, the proposed structure applied to the networked control system is evaluated via experiments.

T. Namerikawa and J. Miyakawa are with Division of Electrical Engineering and Computer Science, Graduate School of Natural Science and Technology, Kanazawa University, Kakuma, Kanazawa 920-1192, JAPAN. toru@t.kanazawa-u.ac.jp
First, a design scheme for the GIMC structure and Smith Predictor are shown. Then we propose a new GIMC structure with Smith Predictor. Next, the magnetic suspension system is described. Since the Smith Predictor cannot be applied to unstable systems, this system is stabilized by a PD controller. The stabilized system is defined as a new augmented plant. We design two controllers using $\mathscr{H}_{\infty}$ mixed sensitivity problems for the new augmented plant, and design the control system of a new GIMC structure with the Smith Predictor based on these controllers. Finally, the proposed structure can achieve both performance and robustness specifications in control experiments for a time-delayed mechatronic system. In addition, the proposed structure can be applied to a virtual networked control system [9] and its effectiveness is shown by several control experiments.

\section{GIMC Structure With SMith Predictor}

\section{A. GIMC Structure}

Let $P(s)$ be a nominal plant model of plant $\tilde{P}(s)$ and $K_{0}(s)$ be a stabilizing controller for $P(s)$. Suppose that $P$ and $K_{0}$ have left coprime factorizations expressed by (1).

$$
P(s)=\tilde{M}(s)^{-1} \tilde{N}(s), \quad K_{0}(s)=\tilde{V}(s)^{-1} \tilde{U}(s)
$$

It is well known that all stabilizing controllers $K(s)$ for $P(s)$ can be expressed in (2) and (3) by using a free-parameter $Q(s) \in R H_{\infty}$

$$
\begin{array}{r}
K(s)=(\tilde{V}(s)-Q(s) \tilde{N}(s))^{-1}(\tilde{U}(s)+Q(s) \tilde{M}(s)) \\
\operatorname{det}(\tilde{V}(\infty)-Q(\infty) \tilde{N}(\infty)) \neq 0 .
\end{array}
$$

GIMC structure is based on this idea and it is shown in Fig. 1. This has an outer feedback $\operatorname{loop}\left(K_{0}(s)=\tilde{V}(s)^{-1} \tilde{U}(s)\right)$ and an internal feedback loop. It is a kind of a extention of the IMC(Internal Model Control) by introducing an outer feedback controller. Note that the reference signal $\operatorname{ref}(t)$ in Fig. 1 enters into the stabilizing controller $K$ in the GIMC

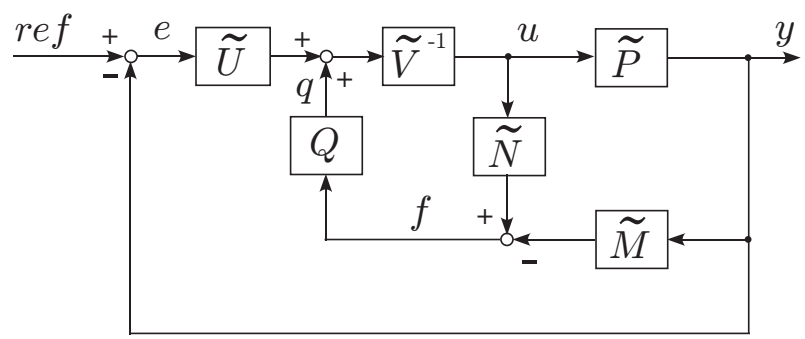

Fig. 1. GIMC Structure 
structure, but stability of system does not change from $K(s)$ because a transfer function from $y(t)$ to $u(t)$ is same with $K(s)=(\tilde{V}(s)-Q(s) \tilde{N}(s))^{-1}(\tilde{U}(s)+Q(s) \tilde{M}(s))$. The freeparameter $Q(s) \in R H_{\infty}$ can be chosen within (3) and $K(s)$ is a set of the stabilizing controllers. We assume that $Q(s)$ is fixed in the following, that means $K(s)$ is fixed by the specified $Q(s)$.

GIMC structure can achieve both high performance and high robustness specifications because it can utilize both controllers $K_{0}(s)$ and $K(s)$ by switching them, it depends on an internal signal $f(s)$. The internal signal $f(s)$ can be expressed in (4) [3].

$$
f(s)=\tilde{N}(s) u(s)-\tilde{M}(s) y(s)
$$

This signal $f(s)$ is an error of an estimated signal and an actual signal. Consider two cases which are $\tilde{P}(s)=P(s)$ and $\tilde{P}(s) \neq P(s)$.

$$
\begin{aligned}
\tilde{P}(s)= & P(s): \\
& f(s)=0 \text { if there are no model uncertainties, distur- } \\
& \text { bance or faults, then } q(s)=0 . \text { The control system } \\
& \text { is controlled by } K_{0}(s)=\tilde{V}(s)^{-1} \tilde{U}(s) . \\
\tilde{P}(s) \neq & P(s): \\
& f(s) \neq 0 \text { if there are either model uncertainties or } \\
& \text { disturbance or faults, then the inner loop is active } \\
& \text { because } q(s) \neq 0 . \text { The feedback system is controlled } \\
& \text { by } K(s)=(\tilde{V}(s)-Q(s) \tilde{N}(s))^{-1}(\tilde{U}(s)+Q(s) \tilde{M}(s)) .
\end{aligned}
$$

GIMC structure can switch two controllers which are $K_{0}(s)$ and $K(s)$ using the internal signal $f(s)$ in the above way. This switching characteristic gives a desired control property to the system. The high performance controller $K_{0}(s)$ is applied to the nominal $\operatorname{model}(f(s)=0)$ and the high robustness controller $K(s)$ is applied to the perturbed $\operatorname{plant}(f(s) \neq 0)$.

The design procedure of GIMC structure is given by the following three steps.

\section{Controller Design Step[2]}

Step 1. Design a high performance controller $K_{0}(s)$ for the nominal model $P(s)$.

Step 2. Design a high robust controller $K(s)$ for the perturbed model $\tilde{P}(s)$.

Step 3. Construct an internal controller $Q(s)$ based on the

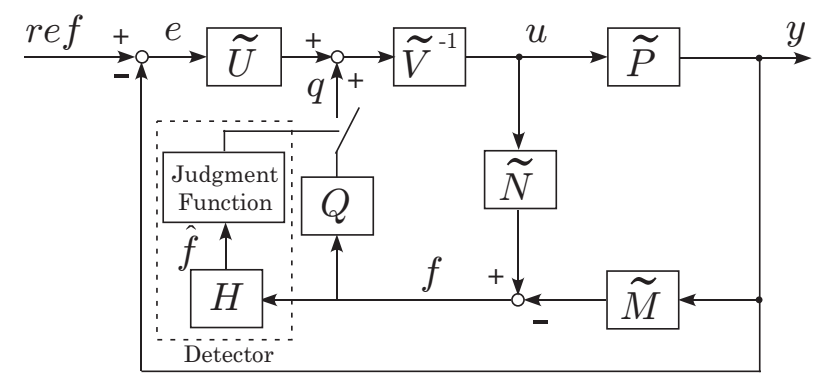

Fig. 2. GIMC Structure with Detector and Switch following equation.

$$
Q(s)=\tilde{V}(s)\left(K(s)-K_{0}(s)\right)(\tilde{N}(s) K(s)+\tilde{M}(s))^{-1}
$$

The internal controller $Q(s)$ is not used in the nominal model then GIMC structure is controlled by only $K_{0}(s)$, and the internal controller $Q(s)$ is activated for the perturbed plant. This means the GIMC structure is controlled by $K(s)$.

\section{Implementation of GIMC-based Switching Controller}

Generally it is impossible to construct a completely accurate plant model such as $\tilde{P}(s)=P(s)$, then $K(s)$ is applied even for the nominal plant because $\tilde{P}(s) \simeq P(s)$ in nominal mode.

Consider GIMC structure with a detector and a switch in the internal loop as shown in Fig.2. This structure makes the high performance controller $K_{0}(s)$ work even if there exists a small perturbation $\tilde{P}(s) \simeq P(s)$. That means the high performance controller $K_{0}$ can be applied to a slightly perturbed nominal model.

In this GIMC structure for implementation, a switching timing and its decision is judged by a signal $\hat{f}(s)$ which is an output of a function $H(s)$. The signal $\hat{f}(s)$ is expressed in (6) and the function $H(s)$ is a filter of the signal $f(s)$ to judge a current mode of the plant.

$$
\hat{f}(s)=H(s)(\tilde{N}(s) u(s)-\tilde{M}(s) y(s))
$$

A judgment index $J_{t h}$ of the nominal and the robust modal is a magnitude of the signal $\hat{f}(s)$ in (7).

The index $J_{t h}$ is utilized to decide a model among the multiple candidates of the plant models. If $\hat{f}(s)<J_{t h}$ then switch is OFF which means the candidate of the nominal plant is selected and if $\hat{f}(s)>J_{t h}$ then the switch is ON.

$$
J_{t h}=\max _{\Delta=0, u}|\hat{f}(s)|, \quad \tilde{P}=P(I+\Delta)
$$

\section{B. Smith Predictor}

Smith Predictor may call Smith method, and this is well known design method of time-delayed system. This system is shown in Fig.3. $P(s) e^{-\tau s}$ is stable time-delayed system and $P(s)$ is stable rational transfer function.

Model plant $P$ in the internal feedback loop predicts an output after delay time. A control input to the plant and delay $\tilde{P}(s) e^{-\hat{\tau} s}$ is decided to base on this predictive output. In outer feedback loop, an output of the plant after delay time

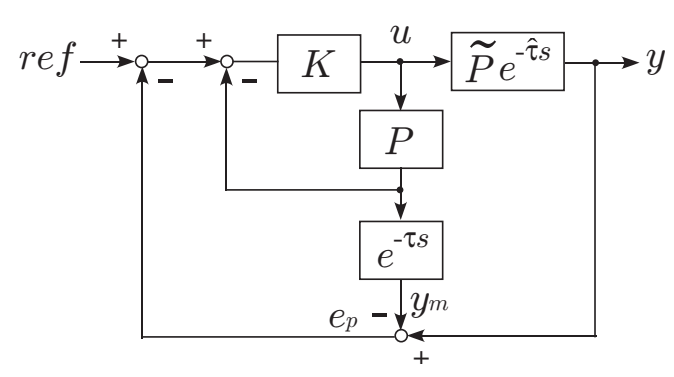

Fig. 3. Smith Predictor 


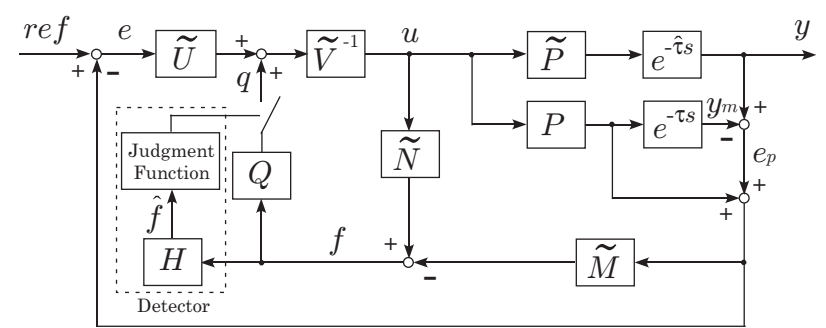

Fig. 4. GIMC Structure with Smith Predictor

is canceled by an output of the model plant $P(s) e^{-\tau s}$. In other words, this structure eliminates an effect of the output after delay time to the predictive control.

\section{GIMC Structure with Smith Predictor}

The structure which can achieve both performance and robustness specifications in the time-delayed system is shown in Fig.4. The proposed control structure in Fig.4 is a combination of GIMC structure and Smith Predictor in Figs. 2 and 3. An output of the plant after delay time is canceled by an output of the model plant, and the system is controlled with the predictive output. The controller of this system is GIMC structure. So this structure can achieve both performance and robustness specifications.

\section{System Configuration AND Modeling}

The controlled plant in this research is a magnetic suspension system[10] shown in Fig.5 where $m$ : mass of iron ball, $f_{\text {mag }}(t)$ : electromagnetic force, $x(t)$ : displacement, $v(t)$ : input voltage, $i(t)$ : current, respectively.

The equation of motion is expressed by (8) and an electromagnetic force is given by (9).

$$
\begin{gathered}
m \frac{d^{2} x(t)}{d t^{2}}=m g-f_{\mathrm{mag}}(t) \\
f_{\mathrm{mag}}(t)=k\left(\frac{i(t)}{x(t)+x_{0}}\right)^{2}
\end{gathered}
$$

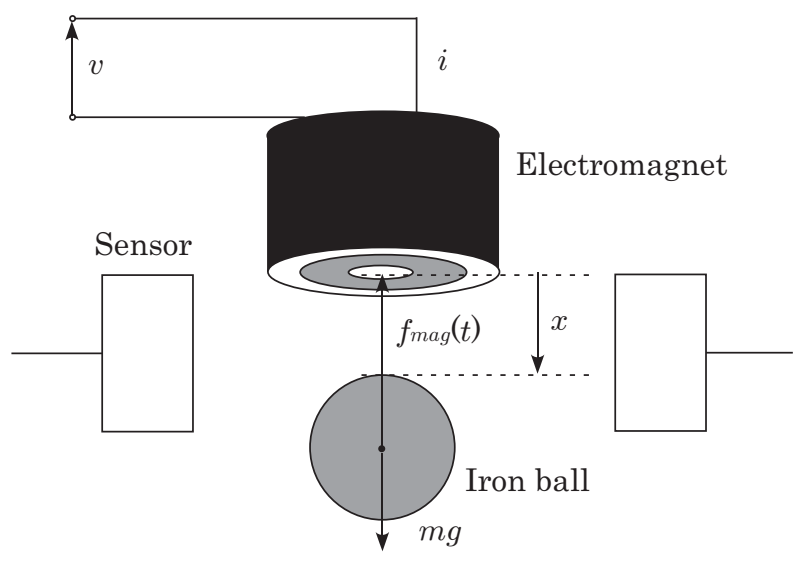

Fig. 5. Magnetic Suspension System
TABLE I

Model Parameters

\begin{tabular}{|c||c|}
\hline$m$ & $0.357[\mathrm{~kg}]$ \\
\hline$k$ & $11.641 \times 10^{-4}\left[\mathrm{Nm}^{2} / \mathrm{A}^{2}\right]$ \\
\hline$x_{0}$ & $4.737 \times 10^{-3}[\mathrm{~m}]$ \\
\hline$X$ & $5 \times 10^{-3}[\mathrm{~m}]$ \\
\hline$I$ & $0.53[\mathrm{~A}]$ \\
\hline
\end{tabular}

The coefficients $k$ and $x_{0}$ in (9) are determined by identification experiments. Equation (9) is transformed into (11) by using Taylor series expansion of (10) around the equilibrium point. The variables in (10) are defined as, $X$ :steady gap between the electromagnet and the iron ball, $\delta x(t)$ :displacement from the steady gap, $I$ :steady current of the electromagnet, $\delta i(t)$ :current from steady current.

$$
\begin{array}{r}
x(t)=X+\delta x(t), i(t)=I+\delta i(t) \\
f_{\text {mag }}(t) \simeq k\left(\frac{I}{X+x_{0}}\right)^{2}+k_{i} \delta i(t)-k_{x} \delta x(t) \\
k_{x}=\frac{2 k I^{2}}{\left(X+x_{0}\right)^{3}}, k_{i}=\frac{2 k I}{\left(X+x_{0}\right)^{2}}
\end{array}
$$

Redefine $x(t)=\delta x(t)$ and $i(t)=\delta i(t)$, then state-space equation is given as (12), and transfer function $P_{0}$ is given as (13). $P_{0}$ is an unstable system from (13). The model parameters are shown in Table I.

$$
\begin{gathered}
\dot{\boldsymbol{x}}=\left[\begin{array}{cc}
0 & 1 \\
K_{x} & 0
\end{array}\right] \boldsymbol{x}+\left[\begin{array}{c}
0 \\
-K_{i}
\end{array}\right] u \\
y=\left[\begin{array}{ll}
1 & 0
\end{array}\right] \boldsymbol{x} \\
P_{0}(s)=\frac{-K_{i}}{s^{2}-K_{x}} \\
\boldsymbol{x}=\left[\begin{array}{cc}
x & \dot{x}
\end{array}\right]^{T}, K_{x}=\frac{k_{x}}{m}, K_{i}=\frac{k_{i}}{m}, y=x, u=i
\end{gathered}
$$

\section{Control System Design}

\section{A. Stabilization of Plant}

A stabilized plant $P_{0}$ by a PD controller is defined as a new augmented plant $P$. Because Smith Predictor cannot be applied to unstable systems. The PD controller $K_{P D}$ is given as (14), and a structure of the new stabilized augmented plant $P$ is shown in Fig.6.

$$
K_{P D}(s)=K_{P}+\frac{K_{D} s}{1+1 / K_{N} s}
$$

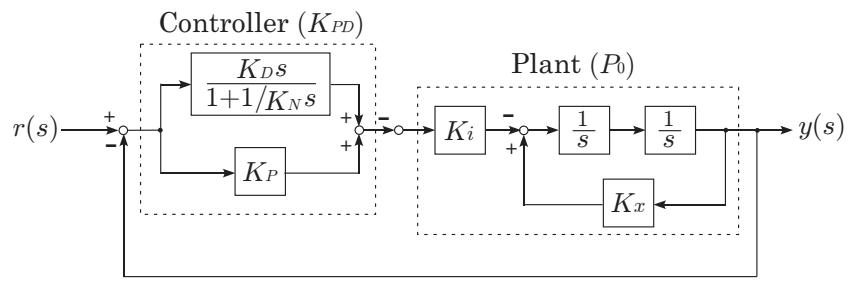

Fig. 6. New Augmented Plant $P$ 
Parameters of this controller are chosen in the following.

$$
K_{P}=180, K_{D}=6, K_{N}=800
$$

A purpose of this controller is to stabilize the plant $P_{0}$, hence the performance is not fully considered.

\section{B. Control system Design}

The design step of controllers is already mentioned in the Section II-A. At first, we design two controllers which are a nominal controller $K_{0}(s)$ and a robust controller $K(s)$ using $\mathscr{H}_{\infty}$ mixed sensitivity problem, respectively on Step 1, 2.

The $\mathscr{H}_{\infty}$ mixed problem is a design problem to find a controller which satisfies the condition (16), where $S(s)$ :sensitivity function, $T(s)$ :complementarity sensitivity function, $W_{S}(s)$ :weighting function for sensitivity function, $W_{T}(s)$ :weighting function for complementarity sensitivity function.

$$
\left\|\begin{array}{c}
W_{S}(s) S(s) \\
W_{T}(s) T(s)
\end{array}\right\|_{\infty}<1
$$

The weighting functions chosen for $K_{0}(s)$ and $K(s)$ are written in (17), (18), respectively.

$$
\begin{aligned}
W_{S_{K_{0}}}(s) & =\frac{20}{s+0.01} \\
W_{T_{K_{0}}}(s) & =1 \times 10^{-4} \times(s+0.02)(s+0.1) \\
W_{S_{K}}(s) & =\frac{10}{s+0.01}, \\
W_{T_{K}}(s) & =1 \times 10^{-3} \times(s+0.02)(s+80)
\end{aligned}
$$

$W_{S_{K_{0}}}$ and $W_{T_{K_{0}}}$ in (17) is used to design $K_{0}(s)$, and $W_{S_{K}}$ and $W_{T_{K}}$ in (18) is used to design $K(s)$, respectively. It is wellknown that there exists a constraint $S(s)+T(s)=I$. Then $W_{S}(s)$ should be selected to have high gain if the designed controller should have high performance, on the other hand, $W_{T}(s)$ should be selected to have high gain if the controller should have high robustness.

In these steps, $K_{0}(s)$ and $K(s)$ are designed to let them have high performance and high robustness respectively. The frequency responses of two controllers are shown in Fig.7, where a solid line shows $K_{0}(s)$, a dashed line shows $K(s)$.

Finally, we construct the internal controller $Q(s)$ by using $K_{0}(s)$ and $K(s)$ based on Step 3. In order to construct $Q(s)$ by using (5), coprime factorizations of plant $P(s)$ and $K_{0}(s)$ are necessary. Suppose that state-space representations of $K_{0}(s)$ and $P(s)$ are given as

$$
P=\left[\begin{array}{l|l}
A & B \\
\hline C & D
\end{array}\right], \quad K_{0}=\left[\begin{array}{c|c}
A_{k} & B_{k} \\
\hline C_{k} & D_{k}
\end{array}\right]
$$

and $(A, B)$ is controllable, $(C, A)$ is observable, $\left(A_{k}, B_{k}\right)$ is controllable and $\left(C_{k}, A_{k}\right)$ is observable.

The coprime factorizations of $P(s)$ and $K_{0}(s)$ are given by (20) and (21) respectively[11]. Note that $L$ and $L_{k}$ stabilize $A+L C$ and $A_{k}+L_{k} C_{k}$, respectively. The eigenvalues are in
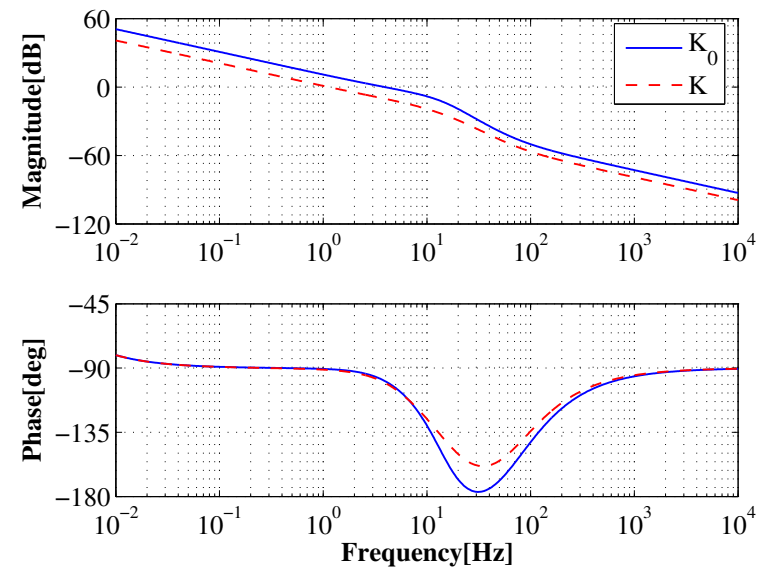

Fig. 7. Bode Diagram of Controllers

TABLE II

Perturbed PARAmeters

\begin{tabular}{|c||c|c|}
\hline & $\begin{array}{c}\text { Nominal } \\
\text { Parameters }\end{array}$ & $\begin{array}{c}\text { Perturbed } \\
\text { Parameters }\end{array}$ \\
\hline \hline$\tau[\mathrm{s}]$ & 0.1 & 0.1 \\
\hline$\hat{\tau}[\mathrm{s}]$ & 0.1 & 0.15 \\
\hline
\end{tabular}

(22) and (23).

$$
\begin{aligned}
{\left[\begin{array}{cc}
\tilde{N} & \tilde{M}
\end{array}\right] } & =\left[\begin{array}{c|cc}
A+L C & B+L D & L \\
\hline C & D & I
\end{array}\right] \\
{\left[\begin{array}{cc}
\tilde{V} & \tilde{U}
\end{array}\right] } & =\left[\begin{array}{c|cc}
A_{k}+L_{k} C_{k} & L_{k} & B_{k}+L_{k} D_{k} \\
\hline C_{k} & I & D_{k}
\end{array}\right] \\
\lambda(A+L C) & =\{-100,-110,-260\} \\
\lambda\left(A_{k}+L_{k} C_{k}\right) & =\{-300,-310,-400,-410\}
\end{aligned}
$$

\section{EXPERIMENTAL EVALUATION}

\section{A. Evaluation of Control Performance}

The first objective of experiments is to evaluate a control performance of the controller $K_{0}=\tilde{V}^{-1} \tilde{U}$ and $K=(\tilde{V}-$ $Q \tilde{N})^{-1}(\tilde{U}+Q \tilde{M})$ in Fig.4. Step responses of the nominal parameters in Table II are shown in Fig.8. The scale of this step reference signal is $1[\mathrm{~mm}]$, and this step is added to the plant at $0.1[\mathrm{~s}]$ where a solid line shows $K_{0}(s)$, a dashed line shows $K(s)$. The transient response of $K_{0}$ is better than $K$ in Fig.8, therefore the controller $K_{0}$ has a higher performance than $K$.

\section{B. Evaluation of Stability}

The time response of the controller $K_{0}$ for perturbed parameters in Table II is shown in Fig.9. This response is gradually getting into instability which means the controller $K_{0}$ cannot keep the stability.

When the communication delay in Table II are added to the plant at $0.1[\mathrm{~s}]$, Time responses of the controlled output and the internal signal $q$ of the proposed GIMC structure with Smith Predictor are shown in Figs.10 and 11 GIMC 
structure with Smith Predictor is confirmed to be able to keep the closed-loop stability. Here the filter $H(s)$ and the threshold value $J_{t h}$ in the detector is given by (24).

$$
H(s)=\frac{1}{1+\frac{s}{2 \pi f_{s}}}, \quad f_{s}=2, \quad J_{t h}=3.5 \times 10^{-4} .
$$

The time response in Fig.10 shows a vibration and an instability after adding the communication delay. On the other hand, the time response of GIMC structure with Smith Predictor shows a stable property. The controllers is switched about 0.63[s] from Fig.11.

From these results, GIMC structure with Smith Predictor achieve a high performance if there is no perturbations in model parameters. Furthermore it can keep the stability even if there is an error of the delay between the plant and the model.

\section{Stability Limitation}

Stability limitation for time delay and perturbation between $\hat{\tau}$ and $\tau$ are evaluated via experiments. GIMC structure with Smith Predictor with more than 10[s] delay is still stable if there is no error in time delay. If there exists an error between $\hat{\tau}$ and $\tau$, the maximum allowable perturbation $|\hat{\tau}-\tau|$ is about $0.16[\mathrm{~s}]$. On the other hand, stability limitation of GIMC structure for time delay is about $0.1[\mathrm{~s}]$. Therefore GIMC structure with Smith Predictor is better than GIMC structure itself.

\section{Vi. Application to Virtual Networked Control SYSTEM}

The proposed structure is applied to a virtually networked control system as shown in Fig.12, where $\hat{\tau}_{\mathrm{cp}}$ : communication delay between the controller and the plant, $\hat{\tau}_{\mathrm{pc}}$ : the communication delay between the plant and the controller, respectively. The virtual network system is constructed in a dSPACE digital control system. Here the communication delay are given as $\tau_{\mathrm{cp}}=\tau_{\mathrm{pc}}=0.32[\mathrm{~s}]$ and $\hat{\tau}_{\mathrm{cp}}=\hat{\tau}_{\mathrm{pc}}=0.37[\mathrm{~s}]$. Then time responses of the output and the internal signal $q$ are shown in Figs.13 and 14. Here the filter $H(s)$ in the detector is given by (24), and the threshold value for the judgment is decided as $J_{t h}=3.0 \times 10^{-4}$. The time response in Fig. 13 shows a vibration and an instability after changing the communication delay. But the time response shows that the vibration is finally decreased and the stability is kept as in Fig.10. We can see that the controllers are switched around 2.5[s] from Fig.14.

It has been shown that the proposed structure can be applied to networked control system from these results as same as the case of the Section V-B.

\section{CONCLUSION}

We have proposed a new GIMC structure with Smith Predictor, and the proposed structure has achieved both high performance and high robustness for a time-delayed unstable mechatronic system. GIMC structure is a structure which can achieve both high performance and high robustness specifications, and Smith Predictor is a control method considering communication delay.

The proposed structure was applied to a magnetic suspension system then it was shown that the structure could achieve high performance if the parameters were not changed, and the structure could keep the stability if there was an error between a plant and a model which a nominal controller could not keep the stability. In addition, the proposed structure was applied to the networked control system and its effectiveness of the proposed approach was shown by several control experiments.

\section{REFERENCES}

[1] K. Zhou and J. C. Doyle, Essential of Robust Control, Prentice Hall, 1998.

[2] K. Zhou, "A Natural Approach to High Performance Robust Control: Another Look at Youla Parameterization," Proceedings of SICE Annual Conference, pp. 869-874, 2004.

[3] K. Zhou and Z. Ren, "A New Controller Architecture for High Performance, Robust, and Fault-Tolerant Control," IEEE Transaction on Automatic Control, vol. 46, no. 10, pp. 1613-1618, 2001.

[4] D. U. Campos-Delgado and K. Zhou, "Reconfigurable Fault-Tolerant Control Using GIMC Structure," IEEE Transactions on Automatic Control, vol. 48, no. 5, pp. 832-838, 2003.

[5] D. U. Campos-Delgado, S. M. Martinez and K. Zhou, "Integrated Fault Tolerant Scheme with Disturbance Feedforward," Proceeding of the American Control Conference, pp. 1799-1804, 2004.

[6] T. Namerikawa and H. Maruyama, "High Performance Robust Control of Magnetic Suspension Systems Using GIMC Structure," Proceeding of the American Control Conference, pp. 4582-4587, 2006.

[7] O. J. M. Smith, "A Controller to Overcome Dead Time," ISA J., 6, pp. 28-33, 1959.

[8] N. Abe and Y. Hirata, "Introduction of Time Delay Systems - Transfer Function Approach-," Journal of The Society of Instrument and Control Engineers, 44-11, pp. 799-804, 2005. (in Japanese)

[9] Y. Tipsuwan and M. Chow, "Control Methodologies in Networked Control Systems," Control Engineering Practice, vol. 11, pp. 1099$1111,2003$.

[10] M. Fujita, T. Namerikawa, F. Matsumura and K. Uchida, $\mu$-Synthesis of an Electromagnetic Suspension System, IEEE Trans. on Automatic Control, Vol.40, No.3, pp. 530-536, 1995

[11] T.T. Tay I. Mareels and J.B. Moore, High Performance Control, Birkhauser, 1998

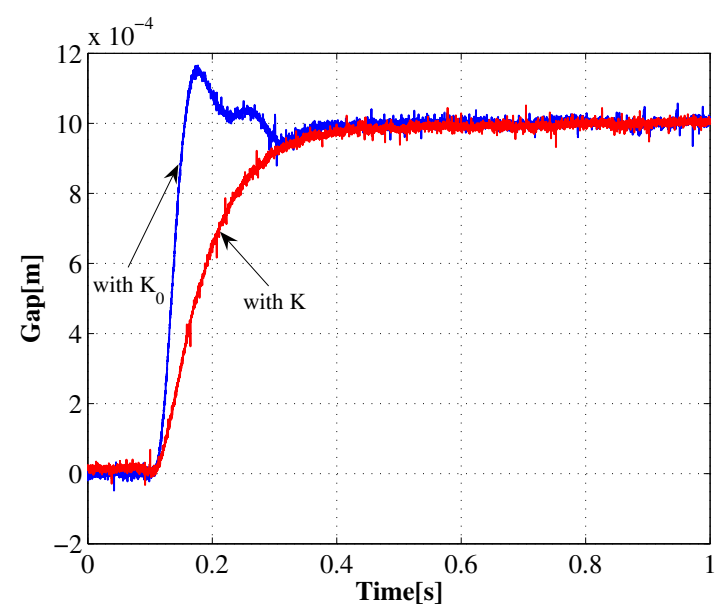

Fig. 8. Step responses with Nominal Parameters 


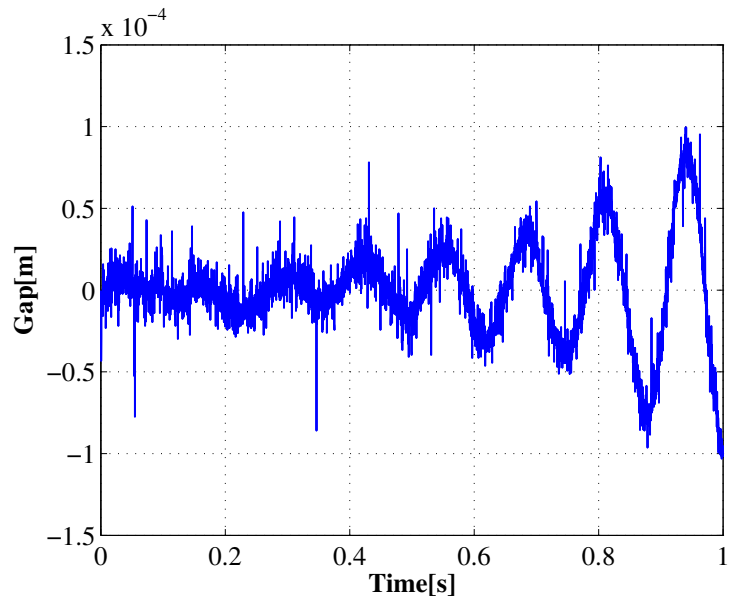

Fig. 9. Time response of $K_{0}$ for Perturbed Parameters

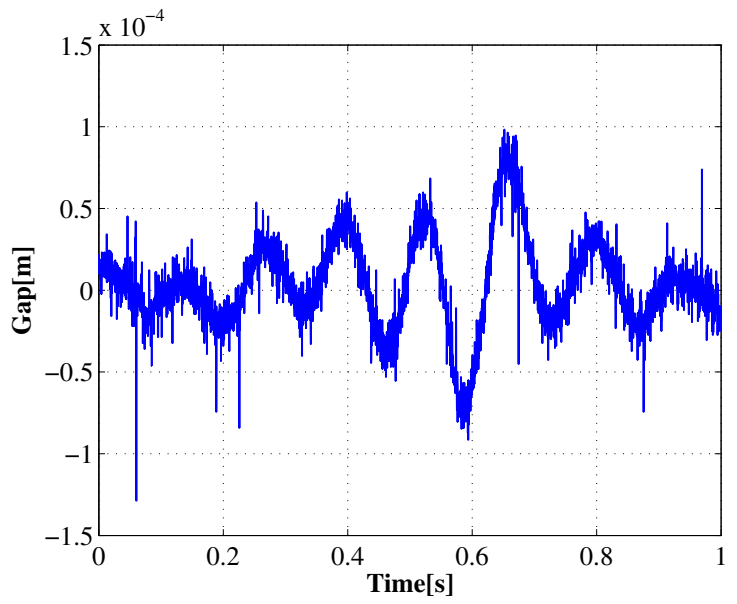

Fig. 10. Time Responses of GIMC with Smith Predictor for Perturbed Parameters

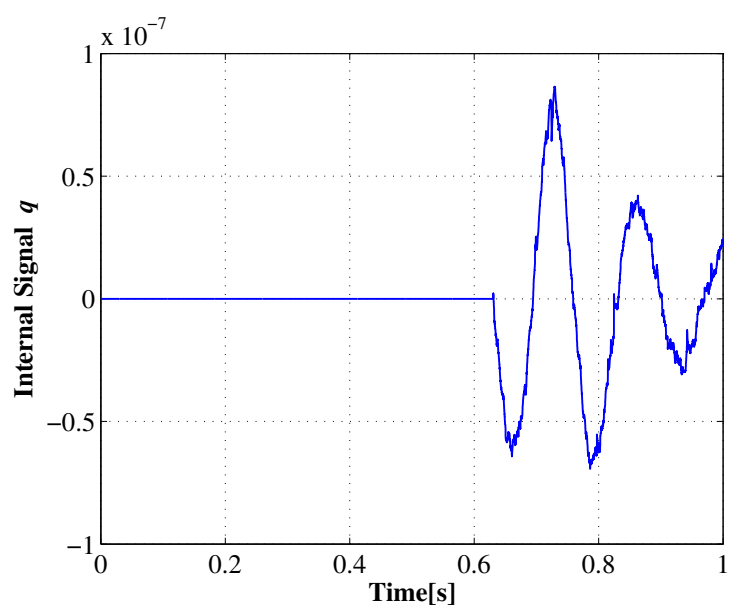

Fig. 11. Internal Signal $q$ of GIMC with Smith Predictor for Perturbed Parameters

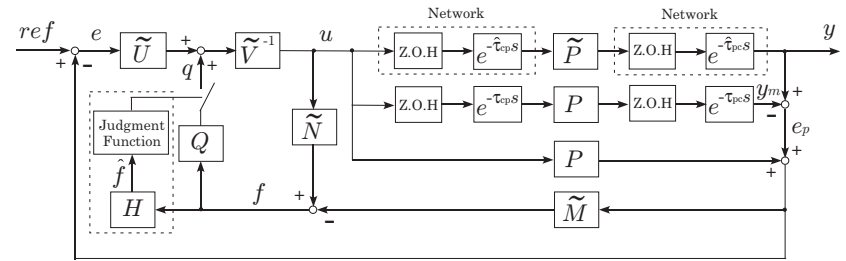

Fig. 12. Networked Control System

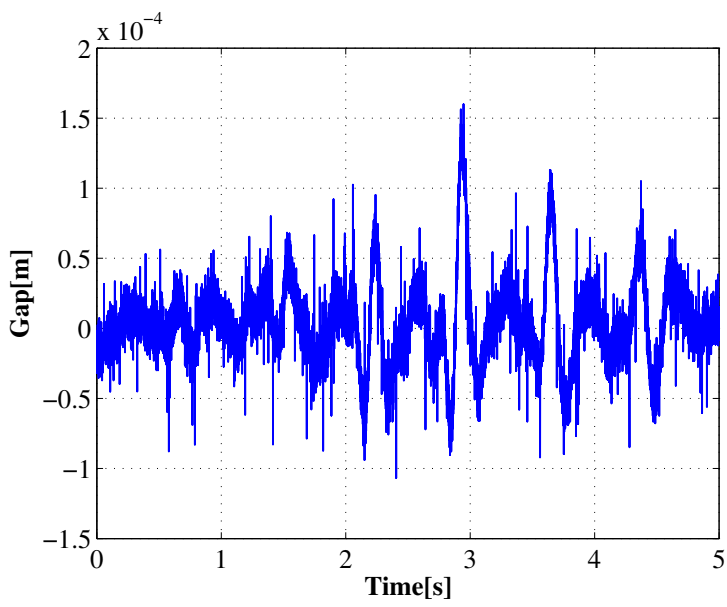

Fig. 13. Time Responses of Networked Control System

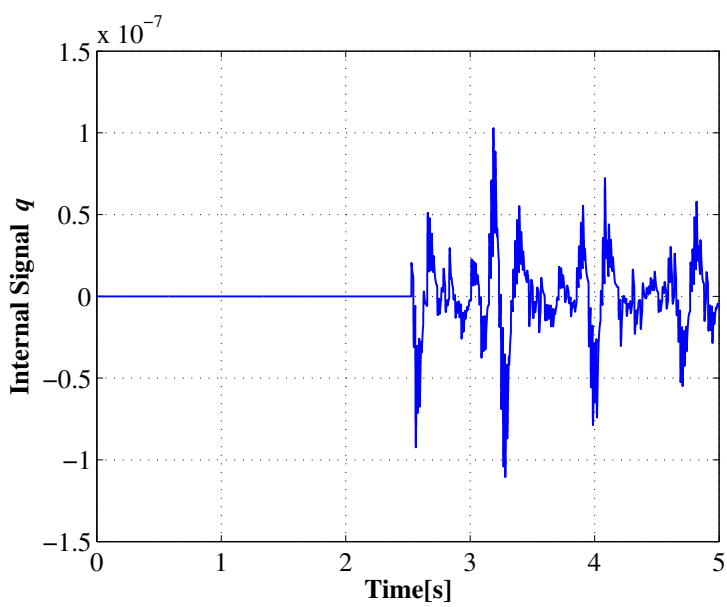

Fig. 14. Internal Signal $q$ of Networked Control System 